\title{
Review Article \\ The Beneficial Effects of Quercetin, Curcumin, and Resveratrol in Obesity
}

\author{
Yueshui Zhao, ${ }^{1}$ Bo Chen, ${ }^{2}$ Jing Shen, ${ }^{1}$ Lin Wan, ${ }^{3}$ Yinxin Zhu, ${ }^{4}$ Tao Yi, ${ }^{5}$ and Zhangang Xiao ${ }^{1,6}$ \\ ${ }^{1}$ Laboratory of Molecular Pharmacology, Department of Pharmacology, School of Pharmacy, Southwest Medical University, Luzhou, \\ Sichuan, China \\ ${ }^{2}$ Experiment Center for Medical Science Research, Kunming Medical University, Kunming, Yunnan, China \\ ${ }^{3}$ Department of Hematology and Oncology, The Children's Hospital of Soochow, Jiangsu, China \\ ${ }^{4}$ Department of Gastroenterology, The Third Affiliated Hospital of Soochow University, Changzhou, Jiangsu, China \\ ${ }^{5}$ School of Chinese Medicine, Hong Kong Baptist University, Kowloon, Hong Kong \\ ${ }^{6}$ Key Laboratory of Medical Electrophysiology of Ministry of Education, School of Pharmacy, Southwest Medical University, Luzhou, \\ Sichuan, China
}

Correspondence should be addressed to Zhangang Xiao; xzg555898@hotmail.com

Received 7 April 2017; Revised 20 July 2017; Accepted 2 August 2017; Published 24 August 2017

Academic Editor: Lin-sen Qing

Copyright (C) 2017 Yueshui Zhao et al. This is an open access article distributed under the Creative Commons Attribution License, which permits unrestricted use, distribution, and reproduction in any medium, provided the original work is properly cited.

Over the past two decades, obesity has been one of the major public health concerns in most countries. In the search for new molecules that could be used for the treatment of obesity, good perspectives have been opened up for polyphenols, a class of natural bioactive phytochemicals. Experimental and limited clinical trial evidence supports that some polyphenols such as quercetin, curcumin, and resveratrol have potential benefit functions on obesity treatment. This brief review focuses on the main functions of the above-named polyphenols on adipose tissue. These polyphenols may play beneficial effects on adipose tissue under obese condition by alleviating intracellular oxidative stress, reducing chronic low-grade inflammation, inhibiting adipogenesis and lipogenesis, and suppressing the differentiation of preadipocytes to mature adipocytes.

\section{Introduction}

Over the past decades, obesity has been one of the major public health threats in most developed countries and in an increasing number of developing countries [1]. Obesity is caused by the imbalance between energy intake and expenditure, which promotes the hypertrophy of adipocytes and results in adipose tissue dysfunction [2]. It is well known that obesity is a strong risk factor for type 2 diabetes mellitus (T2DM) and cancer, and T2DM is linked to the development of cardiovascular diseases, such as hypertension and atherosclerosis [3, 4]. Furthermore, obesity was associated with higher disability rates and mortality rates in the elderly [5]. A better understanding of the molecular basis of obesity will lead to establish strategies for prevention and treatment of obesity.
Adipose tissue is composed of many kinds of cell types, including adipocytes, macrophages, endothelial cells, and stem cells. In addition, as the major energy storage organ, adipose tissue also is a very important endocrine organ [6]. To maintain the function on energy regulation, adipose tissue produces adipokines, such as adiponectin and leptin, and proinflammatory cytokines, such as tumor necrosis factor(TNF-) $\alpha$ and interleukin- (IL-) $1 \beta$ [6]. Under normal physiological lean state, when the body takes excessive energy, adipose tissue can be rapidly enlarged by increasing the adipocyte size (hypertrophy) and numbers (hyperplasia), which were accompanied by an increase of blood vessels (angiogenesis) to supply more oxygen $\left(\mathrm{O}_{2}\right)$ and nutrients to the whole tissue [7]. However, under pathological obese state, adipose tissue will undergo a process named "adipose tissue remodeling," which was characterized by reduced angiogenesis, 
increased hypoxia levels and extra cellular matrix (ECM) levels, and induced higher levels of immune cell infiltration and subsequently induced a low-grade chronic inflammation. All of these pathological events will lead to adipocyte dysfunction, cell death, and systemic insulin resistance [7].

There are two types of adipose tissue, white adipose tissue and brown adipose tissue. The excess energy was mainly stored in the white adipose tissue in the form of triglycerides (TAGs). The function of brown adipose tissue is to directly transfer energy from nutrients to heat by uncoupling protein (UCP) 1, which mediates uncoupling of oxidative phosphorylation from ATP synthesis (conferred thermogenesis) [8-10]. In vitro and in vivo studies demonstrated that the activation of brown adipocytes is an effective and efficient way for excess energy metabolism [11-15]. Human studies showed that activation of brown-like adipocytes is a potential way to counteract obesity $[12,13,16-19]$.

Oxidative stress is referred to an event resulting from the imbalance between the intracellular oxidation system and reduction system, the redox system [20]. The imbalance between oxidant and antioxidant enzymes/substrates will result in a series of oxidation-reduction reactions, which will subsequently induce cytotoxicity by inducing cellular stress responses and stimulating cell death [21]. A series of studies have revealed that oxidative stress is related to the development of obesity. Excess levels of reactive oxygen species (ROS) might lead to the dysfunction of mitochondria by inhibiting respiration process and result in a reduction on the energy expenditure in adipocytes and conversely enhance the energy storage in adipose tissue [22]. Oxidative stress also suppresses the endocrine functions of adipose tissue by disrupting the secretion of adipokines such as adiponectin [23]. Antioxidants can protect cells from oxidative stress by trapping free radicals and restoring cell functions. In recent years, chemical antioxidants derived from natural plants, which are named as "phytochemicals," have gained interest by researchers for preventing and treating diseases, including obesity and obesity-related metabolic diseases [24-29].

Among the phytochemicals studied, researchers pay more attention on polyphenols, which are derived from diet food such as vegetables and fruits, as well as beverages such as juice, coffee, and tea [30-35]. Studies showed that polyphenols such as quercetin, curcumin, and resveratrol exerted beneficial effects on lipid and energy metabolism and potential body weight change. In this review, we will focus on the roles of and the mechanisms of polyphenols including quercetin, curcumin, and resveratrol and on obesity and adipose tissue function.

\section{Quercetin}

Quercetin is the most abundant of flavonoids and is found in vegetables, fruits, tea, and wine [36].

2.1. Effects on Cell Culture Models of Obesity. The first in vitro study investigating the potential antiobesity effect of quercetin on obesity was performed on primary adipocytes. Kuppusamy and Das found that quercetin induced lipolysis of primary rat adipocytes in a dose- and time-dependent manner by increasing cyclic adenosine monophosphate (cAMP) levels and hormone-sensitive lipase (HSL) activity [37]. In addition to the inductive effect on lipolysis, quercetin can also suppress lipogenesis by reducing the incorporation rate of fatty acids into adipocyte triacylglycerols in rat fat pads [38] and by inhibiting the gene expression levels of fatty acid synthase (FAS) and the activity of acetyl-CoA carboxylase (ACC) [39]. Quercetin also can inhibit adipogenesis by decreasing gene expression levels of the key adipogenic factors peroxisome proliferator-activated receptor $\gamma(\operatorname{PPAR} \gamma)$ and CCAAT/enhancer binding protein $\alpha(\mathrm{C} / \mathrm{EBP} \alpha)$ [39]. Recently, using hypertrophied 3T3-L1 adipocyte model, Herranz-López et al. showed that quercetin can rapidly reduce the intracellular ROS levels, which was correlated with the higher levels of quercetin metabolite [40]. Moreover, in human SGBS adipocytes, quercetin can significantly reduce levels of adipokines ANGPTL4, adipsin, and PAI-1 as well as of glycolysis-associated enzymes ENO2, PFKP, and PFKFB4, all of which are associated with obesity and adipose tissue dysfunction [41]. Adipocyte browning is a promising strategy for the prevention of obesity [14, 42-44]. In 3T3-L1 adipocytes, quercetin $(50 \mu \mathrm{M})$ induced the expression of brown adipocyte-specific genes such as UCP-1 and cell death-inducing DNA fragmentation factor-alpha-like effector A (CIDEA) by the activation of AMP-activated protein kinase (AMPK) [45], which is a key checkpoint to control the energy balance in adipocytes by suppressing the activity of ACC; as a result, the levels of lipid in adipocytes were decreased [46].

2.2. Effects on Animal Models of Obesity. Animal studies showed that quercetin can protect mice or rats from highfat diet- (HFD-) induced body weight gain and adipose tissue accumulation [47-49]. In HFD-fed mouse model, Stewart et al. showed that quercetin can transiently increase energy expenditures which may relate to the upregulation of UCP1 [49]. In HFD-fed rat model, quercetin suppressed adipogenesis by reducing the key adipogenic factor $\mathrm{C} / \mathrm{EBP} \alpha$ gene expression levels and reduced lipogenesis by downregulating the gene levels of FAS and ACC [50]. Quercetin also has antiinflammatory effects on adipose tissue. Stewart et al. found that long-time treatment with quercetin can reduce the levels of inflammatory markers IFN $\gamma, \mathrm{TNF} \alpha$, IL- 1 , and IL- 4 in mice [49]. Quercetin suppresses the accumulation and activation of immune cell and improves mitochondrial functions in adipose tissue of HFD-induced obese mice by increasing the levels of oxidative stress-sensitive transcription factor and antioxidant enzymes [51]. Moreover, Dong et al. found that quercetin attenuated mast cell and macrophage infiltration into epididymis adipose tissues (EATs) through the AMPK $\alpha 1$-silent information regulator (SIRT) 1 pathway in HFDfed mice [52]. In Wistar rats, quercetin suppressed the expression of oxidative stress and inflammatory markers, including nuclear factor kappa $\mathrm{B}(\mathrm{NF}-\kappa \mathrm{B})$, nuclear factorrelated factor- (Nrf-) 2, and heme oxygenase- (HO-) 1 [53]. In another study, quercetin ( $10 \mathrm{mg} / \mathrm{kg}$ of body weight) improved the inflammatory status of visceral adipose tissue by suppressing the expression of TNF- $\alpha$ and enhancing the levels of adiponectin, which indicates the recovery of the 
functions of the adipose tissue, in obese Zucker rats, a genetically obese rat model [48].

\subsection{Human Studies and Clinical Trials Using Quercetin to} Treat Obesity. Although many cell culture and animal studies focused on the beneficial effects of quercetin in obesity, there are only a limited number of human studies and clinical trials that have been performed to evaluate the effects of quercetin on obesity treatment. In a 12-week, randomized, doubleblind, placebo-controlled study, Lee et al. demonstrated that quercetin $(100 \mathrm{mg} / \mathrm{day} / \mathrm{subject})$ significantly decreased the total body fat, particularly in the percentage of fat in the arm, and decreased the body mass index (BMI) of overweight or obese subjects [54]. Another study evaluated the effects of quercetin on obesity in overweight-obese subjects with various apolipoprotein $\mathrm{E}$ (APOE) genotypes; the authors reported that quercetin $(150 \mathrm{mg} /$ day/subject $)$ decreased the waist circumference and triacylglycerol concentration [55]. In addition to these findings, one study showed that 12 week of onion extract (quercetin-rich extract) intake decreased body weight, percentage of body fat, and BMI of 10 female university students [56]. However, another study reported that 12-week of onion extract intake has no effect on body fat composition and BMI of the female university students [57], indicating that the experiment period of the study is important for the effects of the onion extracts on body weight change. Currently, there is one clinical trial that is still under phase II stage investigation; the purpose of this study is to investigate whether quercetin changes the absorption of glucose by the body in obese subjects and obese diabetic subjects [58]. Although quercetin suppressed oxidative stress in obese rodent models $[51,53]$, Shanely et al. reported that quercetin has no effect on oxidative stress and antioxidant capacity during a 12-week consuming period of high doses of quercetin (500 or $1000 \mathrm{mg} /$ day/subject) in obese subjects [59]. Future research need to further investigate the bioactive effects and bioavailability of quercetin in the treatment of obesity.

\section{Curcumin}

Curcumin is derived from and is the most bioactive polyphenol in the spice turmeric [60]. Curcumin exerts several biological functions including antioxidation, anti-inflammation, and antiangiogenesis in different organs including adipose tissue [60].

3.1. Effects on Cell Culture Models of Obesity. Curcumin may have a significant effect on adipogenesis. In primary human adipocytes and murine 3T3-L1 adipocytes, curcumin treatment suppressed the expression of adipogenic genes peroxisome proliferator-activated receptor $\gamma(\mathrm{PPAR} \gamma)$ and C/EBP $\alpha$ [61]. In addition to the antiadipogenic effects, curcumin also suppresses the differentiation of preadipocytes to mature adipocytes. Ahn et al. demonstrated that curcumin inhibited 3T3-L1 adipocyte differentiation by inhibiting activities of mitogen-activated protein kinases including ERK, JNK, and p38 [62]. Another report showed that the inhibition effect of curcumin on adipocyte differentiation might have been mediated by the suppression of $\operatorname{PPAR} \gamma$ expression in a dose-dependent manner in human adipocytes [63]. Moreover, curcumin also showed anti-inflammatory effects. Curcumin pretreatment inhibited the secretion of monocyte chemoattractant protein- (MCP-) 1, a proinflammatory cytokine, from 3T3-L1 adipocytes [64].

3.2. Effects on Animal Models of Obesity. Curcumin showed beneficial effects on body weight reduction and energy metabolism. Two weeks of high dietary curcumin supplementation feeding in rats reduced epididymal adipose tissue and increased fatty acid $\beta$-oxidation, indicating the increase of energy expenditure after curcumin treatment [65]. Curcumin also showed anti-inflammatory functions. In HFDinduced obesity and in genetic obesity (ob/ob mice) models, curcumin reduced adipose tissue inflammation by reducing macrophage infiltration into adipose tissue and by increasing adiponectin production $[66,67]$. Curcumin also showed antioxidant effects. Dietary curcumin $(0.2-1 \mathrm{~g} / 100 \mathrm{~g}$ diet $)$ suppressed high-fat-induced lipid accumulation in epididymal adipose tissue [65].

\subsection{Human Studies and Clinical Trials Using Curcumin to} Treat Obesity. Unlike the studies on the effects of curcumin in cells or animals, studies on obese subjects are limited. The first clinical trial using curcumin for obesity treatment was conducted by Mohammadi et al. [68]. In this study, obese subjects were treated with a commercial formulation of curcumin (C3 Complex, $1 \mathrm{~g} /$ day) supplemented with a bioavailability enhancer, piperine $(5 \mathrm{mg} /$ day $)$ for a month. Although there were no changes in weight, body mass index (BMI), or body fat, serum triglyceride levels were significantly decreased after curcumin treatment, indicating the improvement of insulin actions [68]. In another randomized, double-blind, crossover trial, Ganjali and Sahebkar showed that 30-day treatment of C3 Complex $(500 \mathrm{mg}$ /day) plus piperine $(5 \mathrm{mg} /$ day) reduced serum levels of inflammatory cytokines IL- $1 \beta$ and IL-4 of obese individuals [69], indicating the anti-inflammatory activity of curcumin in obesity therapy. Moreover, oral curcumin supplementation $(1 \mathrm{~g} /$ day for 30 days) was effective in reducing oxidative stress burden in obese individuals [70].

Although curcumin has been used for clinical trials in obesity treatment, the multifaceted pharmacological nature of curcumin and its pharmacokinetics and the side effects of curcumin in obesity therapy need to be carefully investigated. The recommended maximum daily usage of curcumin is $1 \mathrm{mg} / \mathrm{kg}$ body weight by a joint report of the World Health Organization and the Food and Agriculture Organization [71]. However, a few studies showed that the chronic use of curcumin can cause liver toxicity [72] and high doses of curcumin can induce gastrointestinal upset, inflamed skin, and chest tightness in a phase II trial in patients with advanced pancreatic cancer [73].

\section{Resveratrol}

Resveratrol (3,5,4'-trihydroxytrans-stilbene) is a small polyphenolic compound, which was well known as constituent 
of red grapes, red wine, peanuts, and ground nuts [74, 75]. Resveratrol showed antioxidant and anti-inflammatory actions [76] and showed beneficial effects in preventing the development of many diseases including obesity and diabetes [77].

4.1. Effects on Cell Culture and Ex Vivo Adipose Tissue Culture Models of Obesity. Resveratrol can inhibit adipogenesis by reducing the stability and transcriptional activity of $\operatorname{PPAR} \gamma[78,79]$ and prevent triglyceride accumulation via enhancing the expression of sirtuin1 (Sirt1), which is an important molecular target regulating cellular energy metabolism and mitochondrial homeostasis [80] in 3T3-L1 adipocytes. Moreover, resveratrol enhanced lipolytic activity in human and rat adipocytes; this effect was mediated by $\beta$ adrenergic activation and the induction of cAMP levels [81, 82]. In addition, to enhance lipolysis, resveratrol also can inhibit lipogenesis by downregulating the expression of lipogenic genes in human adipocytes [83]. Kang et al. found that resveratrol pretreatment suppressed secretion of TNF- $\alpha$ and IL-6 from 3T3-L1 adipocytes and inhibited the activation of inflammatory-related proteins such as extracellular receptor-activated kinase (ERK) and NF-kappaB (NF- $\kappa \mathrm{B})$, indicating that resveratrol has anti-inflammatory effects in adipocytes [84]. In human adipocytes, resveratrol reversed IL-1 $\beta$-stimulated expression of proinflammatory adipokines including IL-6, IL-8, monocyte chemoattractant protein(MCP-) 1, and plasminogen activator inhibitor- (PAI-) 1 $[85,86]$. Moreover, reports showed that resveratrol inhibited adipose tissue inflammation by downregulating the protein levels of IL-6, IL-8, MCP-1, and the inflammatory-related adipokine leptin in human adipose tissue in vitro $[86,87]$.

4.2. Effects on Animal Models of Obesity. Dietary treatment of rodents with resveratrol protected mice against HFDinduced body weight gain and obesity by increasing energy expenditure which was partly mediated by stimulating intracellular mitochondrial functions (fatty acid oxidation) in adipose tissue and by the suppression of fatty acid synthesis [88-90] and by inducing brown-like adipocyte formation in white adipose [91-94]. The in vitro anti-inflammatory effect of resveratrol was also confirmed in animal models. In mice, resveratrol attenuated HFD-induced inflammation of WAT by downregulating the protein levels of proinflammatory cytokines TNF- $\alpha$, IFN- $\alpha$, IFN- $\beta$, and IL-6 [89]. In addition, resveratrol reduced adipose tissue macrophage infiltration [95] and prevented the suppression of the production of regulatory $\mathrm{T}$ cells (Tregs, the negative regulators of inflammation) [96] in HFD-induced obese mice. In Zucker rats, resveratrol suppressed the protein levels of IL- 6 and the activity of NF- $\kappa \mathrm{B}$ in adipose tissue by reducing macrophage infiltration [97]. Interestingly, Jimenez-Gomez et al. showed that resveratrol showed similar effects on high-fat-treated adult rhesus monkey model as effects on HFD-induced obese rodent models, suppressed the activation of NF- $\kappa \mathrm{B}$, and decreased the mRNA levels of IL- 6 , TNF- $\alpha$, IL- $1 \beta$, and adiponectin in the visceral adipose tissue of high-fattreated monkey model [98]. Resveratrol also showed antioxidant effect in animal models. Lv et al. found that resveratrol attenuated diet-induced oxidative stress in epididymal white adipose tissue partly by the reduction of Sirt1 and manganese superoxide dismutase (Sod2) levels [99].

4.3. Clinical Trials Using Resveratrol to Treat Obesity. Although several clinical trials that examine the effect of resveratrol on obesity are currently ongoing (see http:// clinicaltrials.gov) or have finished (see Review [100]), none of them were designed specifically to test the effects of resveratrol on body weight change of obese subjects. In a randomized double-blind cross-over study, Timmers et al. showed that $150 \mathrm{mg}$ /day of resveratrol treatment increased energy expenditure, reduced serum inflammatory markers, and decreased adipose tissue lipolysis and plasma fatty acid and glycerol levels of obese men [101]. In another study, Konings et al. investigated the effects of 30 days resveratrol treatment $(150 \mathrm{mg} / \mathrm{day})$ on the adipocyte size and gene expression patterns in obese men. The authors found that resveratrol treatment decreased the size of abdominal subcutaneous adipocytes [102]. However, another report showed that high levels of resveratrol supplementation treatment had no effect on energy expenditure, adipose tissue content, and metabolic events [103]. The reason for the reversed results obtained from the two reports may possibly lie in the administered doses of resveratrol they used for obesity treatment. The latter report used $1500 \mathrm{mg} /$ day for the trial [103]; this dose was ten times of the dose Konings et al. used in the study [102].

\section{Concluding Remarks}

In the search for new molecules that could be used for the treatment of obesity, good perspectives have been opened up for polyphenols. Current knowledge from cell cultures and animal models suggests that polyphenols, including quercertin, curcumin, and resveratrol, play beneficial effects under obese condition potentially by alleviating intracellular oxidative stress, reducing chronic low-grade inflammation, inhibiting adipogenesis and lipogenesis, and suppressing the differentiation of preadipocytes to mature adipocytes. Although investigators have obtained limited results from clinical trials, there is still no sufficient data to support the high-dose and long-term usage of these polyphenols in obesity treatment.

\section{Conflicts of Interest}

The authors declare no conflict of interest.

\section{Authors' Contributions}

Yueshui Zhao and Bo Chen contributed equally to this work.

\section{Acknowledgments}

This study is supported by grants from the National Natural Science Foundation of China (Grant nos. 81503093, 81602166, and 81672444 ) and the Natural Science Foundation of Jiangsu Province (BK2014086). 


\section{References}

[1] Obesity: preventing and managing the global epidemic. Report of a WHO consultation," World Health Organization Technical Report Series, vol. 894, no. i-xii, pp. 1-253, 2000.

[2] K. Sun, J. Tordjman, K. Clement, and P. E. Scherer, "Fibrosis and adipose tissue dysfunction," Cell Metabolism, vol. 18, no. 4, pp. 470-477, 2013.

[3] V. Cifarelli and S. D. Hursting, "Obesity, diabetes and cancer: a mechanistic perspective," International Journal of Diabetology \& Vascular Disease Research, vol. 2015, Supplement 4, 2015.

[4] Y. Matsuzawa, I. Shimomura, T. Nakamura, Y. Keno, K. Kotani, and K. Tokunaga, "Pathophysiology and pathogenesis of visceral fat obesity," Obesity Research, vol. 3, Supplement 2, pp. 187s-194s, 1995.

[5] V. B. Kalish, "Obesity in older adults," Primary Care, vol. 43, no. 1, pp. 137-144, ix, 2016.

[6] S. E. Wozniak, L. L. Gee, M. S. Wachtel, and E. E. Frezza, "Adipose tissue: the new endocrine organ? A review article," Digestive Diseases and Sciences, vol. 54, no. 9, pp. 18471856, 2009.

[7] K. Sun, C. M. Kusminski, and P. E. Scherer, "Adipose tissue remodeling and obesity," The Journal of Clinical Investigation, vol. 121, no. 6, pp. 2094-2101, 2011.

[8] M. Chondronikola, E. Volpi, E. Børsheim et al., "Brown adipose tissue activation is linked to distinct systemic effects on lipid metabolism in humans," Cell Metabolism, vol. 23, no. 6, pp. 1200-1206, 2016.

[9] B. Cannon and J. Nedergaard, "Brown adipose tissue: function and physiological significance," Physiological Reviews, vol. 84, no. 1, pp. 277-359, 2004.

[10] R. Oelkrug, E. T. Polymeropoulos, and M. Jastroch, "Brown adipose tissue: physiological function and evolutionary significance," Journal of Comparative Physiology B, Biochemical, Systemic, and Environmental Physiology, vol. 185, no. 6, pp. 587-606, 2015.

[11] C. E. Varela, A. Rodriguez, M. Romero-Valdovinos et al., "Browning effects of (-)-epicatechin on adipocytes and white adipose tissue," European Journal of Pharmacology, 2017.

[12] B. Thyagarajan and M. T. Foster, "Beiging of white adipose tissue as a therapeutic strategy for weight loss in humans," Hormone Molecular Biology and Clinical Investigation, 2017.

[13] P. C. Dinas, A. Valente, M. Granzotto et al., "Browning formation markers of subcutaneous adipose tissue in relation to resting energy expenditure, physical activity and diet in humans," 2017.

[14] N. J. Song, S. H. Chang, D. Y. Li, C. J. Villanueva, and K. W. Park, "Induction of thermogenic adipocytes: molecular targets and thermogenic small molecules," Experimental \& Molecular Medicine, vol. 49, no. 7, article e353, 2017.

[15] V. Ryu, E. Zarebidaki, H. Elliott Albers, B. Xue, and T. J. Bartness, "Short photoperiod reverses obesity in Siberian hamsters via sympathetically induced lipolysis and browning in adipose tissue," Physiology \& Behavior, 2017.

[16] B. Halpern, M. C. Mancini, and A. Halpern, "Brown adipose tissue: what have we learned since its recent identification in human adults," Arquivos Brasileiros de Endocrinologia e Metabologia, vol. 58, no. 9, pp. 889-899, 2014.

[17] W. D. Marken Lichtenbeltvan, J. W. Vanhommerig, N. M. Smulders et al., "Cold-activated brown adipose tissue in healthy men," The New England Journal of Medicine, vol. 360, no. 15, pp. 1500-1508, 2009.

[18] M. Saito, Y. Okamatsu-Ogura, M. Matsushita et al., "High incidence of metabolically active brown adipose tissue in healthy adult humans: effects of cold exposure and adiposity," Diabetes, vol. 58, no. 7, pp. 1526-1531, 2009.

[19] V. Ouellet, S. M. Labbe, D. P. Blondin et al., "Brown adipose tissue oxidative metabolism contributes to energy expenditure during acute cold exposure in humans," The Journal of Clinical Investigation, vol. 122, no. 2, pp. 545552, 2012.

[20] C. M. Grant, "Metabolic reconfiguration is a regulated response to oxidative stress," Journal of Biology, vol. 7, no. 1, p. 1, 2008.

[21] G. Bjorklund and S. Chirumbolo, "Role of oxidative stress and antioxidants in daily nutrition and human health," Nutrition (Burbank, Los Angeles County, Calif.), vol. 33, pp. 311-321, 2017.

[22] T. Wang, Y. Si, O. S. Shirihai et al., "Respiration in adipocytes is inhibited by reactive oxygen species," Obesity (Silver Spring, Md.), vol. 18, no. 8, pp. 1493-1502, 2010.

[23] M. Matsuda and I. Shimomura, "Roles of oxidative stress, adiponectin, and nuclear hormone receptors in obesityassociated insulin resistance and cardiovascular risk," Hormone Molecular Biology and Clinical Investigation, vol. 19, no. 2, pp. 75-88, 2014.

[24] R. Mopuri and M. S. Islam, "Medicinal plants and phytochemicals with anti-obesogenic potentials: a review," Biomedicine \& Pharmacotherapy = Biomedecine \& Pharmacotherapie, vol. 89, pp. 1442-1452, 2017.

[25] Y. Tang and R. Tsao, "Phytochemicals in quinoa and amaranth grains and their antioxidant, anti-inflammatory, and potential health beneficial effects: a review," Molecular Nutrition \& Food Research, vol. 61, no. 7, 2017.

[26] J. Martel, D. M. Ojcius, C. J. Chang et al., "Anti-obesogenic and antidiabetic effects of plants and mushrooms," Nature Reviews Endocrinology, vol. 13, no. 3, pp. 149-160, 2017.

[27] N. N. Sun, T. Y. Wu, and C. F. Chau, "Natural dietary and herbal products in anti-obesity treatment," Molecules (Basel, Switzerland), vol. 21, no. 10, 2016.

[28] M. Balaji, M. S. Ganjayi, G. E. Hanuma Kumar, B. N. Parim, R. Mopuri, and S. Dasari, "A review on possible therapeutic targets to contain obesity: the role of phytochemicals," Obesity Research \& Clinical Practice, vol. 10, no. 4, pp. 363-380, 2016.

[29] Y. J. Zhang, R. Y. Gan, S. Li et al., "Antioxidant phytochemicals for the prevention and treatment of chronic diseases," Molecules (Basel, Switzerland), vol. 20, no. 12, pp. 2113821156, 2015.

[30] S. S. Deshpande, S. K. Sathe, and D. K. Salunkhe, "Chemistry and safety of plant polyphenols," Advances in Experimental Medicine and Biology, vol. 177, pp. 457-495, 1984.

[31] L. Vamos-Vigyazo, "Polyphenol oxidase and peroxidase in fruits and vegetables," Critical Reviews in Food Science and Nutrition, vol. 15, no. 1, pp. 49-127, 1981.

[32] E. Haslam, T. H. Lilley, Y. Cai, R. Martin, and D. Magnolato, "Traditional herbal medicines-the role of polyphenols," Planta Medica, vol. 55, no. 1, pp. 1-8, 1989.

[33] C. Rice-Evans, "Plant polyphenols: free radical scavengers or chain-breaking antioxidants?," Biochemical Society Symposium, vol. 61, pp. 103-116, 1995. 
[34] J. A. Dominguez Avila, J. Rodrigo Garcia, G. A. Gonzalez Aguilar, and L. A. Rosade la, "The antidiabetic mechanisms of polyphenols related to increased glucagon-like peptide-1 (GLP1) and insulin signaling," Molecules (Basel, Switzerland), vol. 22, no. 6, 2017.

[35] J. Teixeira, D. Chavarria, F. Borges et al., "Dietary polyphenols and mitochondrial function: role in health and disease," Current Medicinal Chemistry, 2017.

[36] R. J. Nijveldt, E. Noodvan, D. E. Hoornvan, P. G. Boelens, K. Norrenvan, and P. A. Leeuwenvan, "Flavonoids: a review of probable mechanisms of action and potential applications," The American Journal of Clinical Nutrition, vol. 74, no. 4, pp. 418-425, 2001.

[37] U. R. Kuppusamy and N. P. Das, "Effects of flavonoids on cyclic AMP phosphodiesterase and lipid mobilization in rat adipocytes," Biochemical Pharmacology, vol. 44, no. 7, pp. 1307-1315, 1992.

[38] T. Motoyashiki, T. Morita, and H. Ueki, "Involvement of the rapid increase in cAMP content in the vanadate-stimulated release of lipoprotein lipase activity from rat fat pads," Biological \& Pharmaceutical Bulletin, vol. 19, no. 11, pp. 14121416, 1996.

[39] J. Ahn, H. Lee, S. Kim, J. Park, and T. Ha, “The anti-obesity effect of quercetin is mediated by the AMPK and MAPK signaling pathways," Biochemical and Biophysical Research Communications, vol. 373, no. 4, pp. 545-549, 2008.

[40] M. Herranz-López, I. Borras-Linares, M. Olivares-Vicente, J. Galvez, A. Segura-Carretero, and V. Micol, "Correlation between the cellular metabolism of quercetin and its glucuronide metabolite and oxidative stress in hypertrophied 3T3-L1 adipocytes," Phytomedicine: International Journal of Phytotherapy and Phytopharmacology, vol. 25, pp. 25-28, 2017.

[41] A. Leiherer, K. Stoemmer, A. Muendlein et al., "Quercetin impacts expression of metabolism- and obesity-associated genes in SGBS adipocytes," Nutrients, vol. 8, no. 5, p. 282, 2016.

[42] C. R. Yeo, M. Agrawal, S. Hoon et al., "SGBS cells as a model of human adipocyte browning: a comprehensive comparative study with primary human white subcutaneous adipocytes," Scientific Reports, vol. 7, no. 1, p. 4031, 2017.

[43] S. Carobbio, V. Pellegrinelli, and A. Vidal-Puig, "Adipose tissue function and expandability as determinants of lipotoxicity and the metabolic syndrome," Advances in Experimental Medicine and Biology, vol. 960, pp. 161-196, 2017.

[44] M. Okla, J. Kim, and K. Koehler, "Dietary factors promoting brown and beige fat development and thermogenesis," Advances in Nutrition (Bethesda, Md.), vol. 8, no. 3, pp. 473-483, 2017.

[45] S. G. Lee, J. S. Parks, and H. W. Kang, "Quercetin, a functional compound of onion peel, remodels white adipocytes to brown-like adipocytes," The Journal of Nutritional Biochemistry, vol. 42, pp. 62-71, 2017.

[46] X. Zhou, J. Xu, Y. Shi, and J. M. Ye, "Discovery of novel antidiabetic drugs by targeting lipid metabolism," Current Drug Targets, vol. 16, no. 12, pp. 1372-1380, 2015.

[47] C. Liang, M. E. Oest, and M. R. Prater, "Intrauterine exposure to high saturated fat diet elevates risk of adult-onset chronic diseases in C57BL/6 mice," Birth Defects Research Part B, Developmental and Reproductive Toxicology, vol. 86, no. 5, pp. 377-384, 2009.
[48] L. Rivera, R. Moron, M. Sanchez, A. Zarzuelo, and M. Galisteo, "Quercetin ameliorates metabolic syndrome and improves the inflammatory status in obese Zucker rats," Obesity (Silver Spring, Md.), vol. 16, no. 9, pp. 2081-2087, 2008.

[49] L. K. Stewart, J. L. Soileau, D. Ribnicky et al., "Quercetin transiently increases energy expenditure but persistently decreases circulating markers of inflammation in C57BL/6J mice fed a high-fat diet," Metabolism: Clinical and Experimental, vol. 57, no. 7, Supplement 1, pp. S39-S46, 2008.

[50] J. Moon, H. J. Do, O. Y. Kim, and M. J. Shin, “Antiobesity effects of quercetin-rich onion peel extract on the differentiation of 3T3-L1 preadipocytes and the adipogenesis in high fat-fed rats," Food and Chemical Toxicology: An International Journal Published for the British Industrial Biological Research Association, vol. 58, pp. 347-354, 2013.

[51] M. Kobori, Y. Takahashi, M. Sakurai et al., "Quercetin suppresses immune cell accumulation and improves mitochondrial gene expression in adipose tissue of diet-induced obese mice," Molecular Nutrition \& Food Research, vol. 60, no. 2, pp. 300-312, 2016.

[52] J. Dong, X. Zhang, L. Zhang et al., "Quercetin reduces obesity-associated ATM infiltration and inflammation in mice: a mechanism including AMPKalpha1/SIRT1," Journal of Lipid Research, vol. 55, no. 3, pp. 363-374, 2014.

[53] S. K. Panchal, H. Poudyal, and L. Brown, "Quercetin ameliorates cardiovascular, hepatic, and metabolic changes in diet-induced metabolic syndrome in rats," The Journal of Nutrition, vol. 142, no. 6, pp. 1026-1032, 2012.

[54] J.-S. Lee, Y.-J. Cha, K.-H. Lee, and J.-E. Yim, “Onion peel extract reduces the percentage of body fat in overweight and obese subjects: a 12 -week, randomized, double-blind, placebo-controlled study," Nutrition Research Practice, vol. 10, no. 2, pp. 175-181, 2016.

[55] M. Pfeuffer, A. Auinger, U. Bley et al., "Effect of quercetin on traits of the metabolic syndrome, endothelial function and inflammation in men with different APOE isoforms," Nutrition, Metabolism, and Cardiovascular Diseases: NMCD, vol. 23, no. 5, pp. 403-409, 2013.

[56] Y. K. Yang and S. P. Kim, "The effect of onion extract intake for 12 weeks on blood lipid and obesity index in obese university women," Korean Journal of Sports Sciences, vol. 22, pp. 955-962, 2013.

[57] K. H. Lee, H. J. Lee, E. J. Park, and K. I. Jeon, "Effect of onion extracts on serum lipid and antioxidant status in healthy university female," Korean Journal of Community Nutrition, vol. 2, 2008.

[58] Trials.gov C, "Investigating the use of quercetin on glucose absorption in obesity, and obesity with type 2 diabetes," 2017, http://clinicaltrials.gov/show/NCT00065676, 2013.

[59] R. A. Shanely, A. M. Knab, D. C. Nieman, F. Jin, S. R. McAnulty, and M. J. Landram, "Quercetin supplementation does not alter antioxidant status in humans," Free Radical Research, vol. 44, no. 2, pp. 224-231, 2010.

[60] A. S. Strimpakos and R. A. Sharma, "Curcumin: preventive and therapeutic properties in laboratory studies and clinical trials," Antioxidants \& Redox Signaling, vol. 10, no. 3, pp. 511-545, 2008.

[61] C. Y. Kim, T. T. Le, C. Chen, J. X. Cheng, and K. H. Kim, "Curcumin inhibits adipocyte differentiation through modulation of mitotic clonal expansion," The Journal of Nutritional Biochemistry, vol. 22, no. 10, pp. 910-920, 2011. 
[62] J. Ahn, H. Lee, S. Kim, and T. Ha, "Curcumin-induced suppression of adipogenic differentiation is accompanied by activation of Wnt/beta-catenin signaling," American Journal of Physiology Cell Physiology, vol. 298, no. 6, pp. C1510C1516, 2010.

[63] M. Gurnell, "Peroxisome proliferator-activated receptor gamma and the regulation of adipocyte function: lessons from human genetic studies," Best Practice \& Research Clinical Endocrinology \& Metabolism, vol. 19, no. 4, pp. 501-523, 2005.

[64] H. M. Woo, J. H. Kang, T. Kawada, H. Yoo, M. K. Sung, and R. Yu, "Active spice-derived components can inhibit inflammatory responses of adipose tissue in obesity by suppressing inflammatory actions of macrophages and release of monocyte chemoattractant protein-1 from adipocytes," Life Sciences, vol. 80, no. 10, pp. 926-931, 2007.

[65] A. Asai and T. Miyazawa, "Dietary curcuminoids prevent high-fat diet-induced lipid accumulation in rat liver and epididymal adipose tissue," The Journal of Nutrition, vol. 131, no. 11, pp. 2932-2935, 2001.

[66] W. Shao, Z. Yu, Y. Chiang et al., "Curcumin prevents high fat diet induced insulin resistance and obesity via attenuating lipogenesis in liver and inflammatory pathway in adipocytes," PLoS One, vol. 7, no. 1, article e28784, 2012.

[67] S. P. Weisberg, R. Leibel, and D. V. Tortoriello, "Dietary curcumin significantly improves obesity-associated inflammation and diabetes in mouse models of diabesity," Endocrinology, vol. 149, no. 7, pp. 3549-3558, 2008.

[68] A. Mohammadi, A. Sahebkar, M. Iranshahi et al., "Effects of supplementation with curcuminoids on dyslipidemia in obese patients: a randomized crossover trial," Phytotherapy Research: PTR, vol. 27, no. 3, pp. 374-379, 2013.

[69] S. Ganjali and A. Sahebkar, "Investigation of the effects of curcumin on serum cytokines in obese individuals: a randomized controlled trial," The Scientific World Journal, vol. 2014, Article ID 898361, 6 pages, 2014.

[70] A. Sahebkar, A. Mohammadi, A. Atabati et al., "Curcuminoids modulate pro-oxidant-antioxidant balance but not the immune response to heat shock protein 27 and oxidized LDL in obese individuals," Phytotherapy Research: PTR, vol. 27, no. 12, pp. 1883-1888, 2013.

[71] Evaluation of certain food additives," WHO Technical Report Series, 891, WHO, Geneva, 2000.

[72] M. Liddle, C. Hull, C. Liu, and D. Powell, "Contact urticaria from curcumin," Dermatitis: Contact, Atopic, Occupational, Drug, vol. 17, no. 4, pp. 196-197, 2006.

[73] N. Dhillon, B. B. Aggarwal, R. A. Newman et al., "Phase II trial of curcumin in patients with advanced pancreatic cancer," Clinical Cancer Research: An Official Journal of the American Association for Cancer Research, vol. 14, no. 14, pp. 4491-4499, 2008.

[74] M. Meydani and S. T. Hasan, "Dietary polyphenols and obesity," Nutrients, vol. 2, no. 7, pp. 737-751, 2010.

[75] U. Stervbo, O. Vang, and C. Bonnesen, "A review of the content of the putative chemopreventive phytoalexin resveratrol in red wine," Food Chemistry, vol. 101, no. 2, pp. 449457, 2007.

[76] J. Burns, T. Yokota, H. Ashihara, M. E. Lean, and A. Crozier, "Plant foods and herbal sources of resveratrol," Journal of Agricultural and Food Chemistry, vol. 50, no. 11, pp. 33373340, 2002.
[77] W. J. Spuyvan der and E. Pretorius, "Is the use of resveratrol in the treatment and prevention of obesity premature?" Nutrition Research Reviews, vol. 22, no. 2, pp. 111-117, 2009.

[78] Z. E. Floyd, Z. Q. Wang, G. Kilroy, and W. T. Cefalu, "Modulation of peroxisome proliferator-activated receptor gamma stability and transcriptional activity in adipocytes by resveratrol," Metabolism, vol. 57, no. 7, Supplement 1, pp. S32-S38, 2008.

[79] S. Rayalam, J. Y. Yang, S. Ambati, M. A. Della-Fera, and C. A. Baile, "Resveratrol induces apoptosis and inhibits adipogenesis in 3T3-L1 adipocytes," Phytotherapy Research: PTR, vol. 22, no. 10, pp. 1367-1371, 2008.

[80] H. Imamura, D. Nagayama, N. Ishihara et al., "Resveratrol attenuates triglyceride accumulation associated with upregulation of Sirt1 and lipoprotein lipase in 3T3-L1 adipocytes," Molecular Genetics and Metabolism Reports, vol. 12, pp. 4450, 2017.

[81] K. Szkudelska, L. Nogowski, and T. Szkudelski, "Resveratrol, a naturally occurring diphenolic compound, affects lipogenesis, lipolysis and the antilipolytic action of insulin in isolated rat adipocytes," The Journal of Steroid Biochemistry and Molecular Biology, vol. 113, no. 1-2, pp. 17-24, 2009.

[82] S. Gomez-Zorita, K. Treguer, J. Mercader, and C. Carpene, "Resveratrol directly affects in vitro lipolysis and glucose transport in human fat cells," Journal of Physiology and Biochemistry, vol. 69, no. 3, pp. 585-593, 2013.

[83] P. Fischer-Posovszky, V. Kukulus, D. Tews et al., "Resveratrol regulates human adipocyte number and function in a Sirt1-dependent manner," The American Journal of Clinical Nutrition, vol. 92, no. 1, pp. 5-15, 2010.

[84] L. Kang, W. Heng, A. Yuan, L. Baolin, and H. Fang, "Resveratrol modulates adipokine expression and improves insulin sensitivity in adipocytes: relative to inhibition of inflammatory responses," Biochimie, vol. 92, no. 7, pp. 789-796, 2010.

[85] J. Olholm, S. K. Paulsen, K. B. Cullberg, B. Richelsen, and S. B. Pedersen, "Anti-inflammatory effect of resveratrol on adipokine expression and secretion in human adipose tissue explants," International Journal of Obesity (2005), vol. 34, no. 10, pp. 1546-1553, 2010.

[86] I. Zagotta, E. Y. Dimova, K. M. Debatin, M. Wabitsch, T. Kietzmann, and P. Fischer-Posovszky, "Obesity and inflammation: reduced cytokine expression due to resveratrol in a human in vitro model of inflamed adipose tissue," Frontiers in Pharmacology, vol. 6, p. 79, 2015.

[87] K. B. Cullberg, J. Olholm, S. K. Paulsen et al., "Resveratrol has inhibitory effects on the hypoxia-induced inflammation and angiogenesis in human adipose tissue in vitro," European Journal of Pharmaceutical Sciences: Official Journal of the European Federation for Pharmaceutical Sciences, vol. 49, no. 2, pp. 251-257, 2013.

[88] S. J. Cho, U. J. Jung, and M. S. Choi, "Differential effects of low-dose resveratrol on adiposity and hepatic steatosis in diet-induced obese mice," The British Journal of Nutrition, vol. 108, no. 12, pp. 2166-2175, 2012.

[89] S. Kim, Y. Jin, Y. Choi, and T. Park, "Resveratrol exerts antiobesity effects via mechanisms involving down-regulation of adipogenic and inflammatory processes in mice," Biochemical Pharmacology, vol. 81, no. 11, pp. 1343-1351, 2011.

[90] M. Lagouge, C. Argmann, Z. Gerhart-Hines et al., "Resveratrol improves mitochondrial function and protects against metabolic disease by activating SIRT1 and PGC-1alpha," Cell, vol. 127, no. 6, pp. 1109-1122, 2006. 
[91] C. R. Ku, Y. H. Cho, Z. Y. Hong et al., "The effects of high fat diet and resveratrol on mitochondrial activity of brown adipocytes," Endocrinology and Metabolism (Seoul, Korea), vol. 31, no. 2, pp. 328-335, 2016.

[92] G. Alberdi, V. M. Rodriguez, J. Miranda, M. T. Macarulla, I. Churruca, and M. P. Portillo, "Thermogenesis is involved in the body-fat lowering effects of resveratrol in rats," Food Chemistry, vol. 141, no. 2, pp. 1530-1535, 2013.

[93] N. Arias, C. Pico, M. Teresa Macarulla et al., "A combination of resveratrol and quercetin induces browning in white adipose tissue of rats fed an obesogenic diet," Obesity (Silver Spring, Md.), vol. 25, no. 1, pp. 111-121, 2017.

[94] S. Wang, X. Liang, Q. Yang et al., "Resveratrol induces brown-like adipocyte formation in white fat through activation of AMP-activated protein kinase (AMPK) alpha1," International Journal of Obesity (2005), vol. 39, no. 6, pp. 967-976, 2015.

[95] B. T. Jeon, E. A. Jeong, H. J. Shin et al., "Resveratrol attenuates obesity-associated peripheral and central inflammation and improves memory deficit in mice fed a high-fat diet," Diabetes, vol. 61, no. 6, pp. 1444-1454, 2012.

[96] B. Wang, J. Sun, X. Li et al., "Resveratrol prevents suppression of regulatory T-cell production, oxidative stress, and inflammation of mice prone or resistant to high-fat diet-induced obesity," Nutrition Research (New York, N.Y.), vol. 33, no. 11, pp. 971-981, 2013.

[97] S. Gomez-Zorita, A. Fernandez-Quintela, A. Lasa, E. Hijona, L. Bujanda, and M. P. Portillo, "Effects of resveratrol on obesity-related inflammation markers in adipose tissue of genetically obese rats," Nutrition (Burbank, Los Angeles County, Calif.), vol. 29, no. 11-12, pp. 1374-1380, 2013.

[98] Y. Jimenez-Gomez, J. A. Mattison, K. J. Pearson et al., "Resveratrol improves adipose insulin signaling and reduces the inflammatory response in adipose tissue of rhesus monkeys on high-fat, high-sugar diet," Cell Metabolism, vol. 18, no. 4, pp. 533-545, 2013.

[99] Z. M. Lv, Q. Wang, Y. H. Chen, S. H. Wang, and D. Q. Huang, "Resveratrol attenuates inflammation and oxidative stress in epididymal white adipose tissue: implications for its involvement in improving steroidogenesis in dietinduced obese mice," Molecular Reproduction and Development, vol. 82, no. 4, pp. 321-328, 2015.

[100] S. Timmers, M. K. Hesselink, and P. Schrauwen, "Therapeutic potential of resveratrol in obesity and type 2 diabetes: new avenues for health benefits?," Annals of the New York Academy of Sciences, vol. 1290, pp. 83-89, 2013.

[101] S. Timmers, E. Konings, L. Bilet et al., "Calorie restriction-like effects of 30 days of resveratrol $\left(\mathrm{resVida}^{\mathrm{TM}}\right.$ ) supplementation on energy metabolism and metabolic profile in obese humans," Cell Metabolism, vol. 14, no. 5, pp. 612-622, 2011.

[102] E. Konings, S. Timmers, M. V. Boekschoten et al., "The effects of 30 days resveratrol supplementation on adipose tissue morphology and gene expression patterns in obese men," International Journal of Obesity (2005), vol. 38, no. 3, pp. 470-473, 2014.

[103] M. M. Poulsen, P. F. Vestergaard, B. F. Clasen et al., "Highdose resveratrol supplementation in obese men. An investigator-initiated, randomized, placebo-controlled clinical trial of substrate metabolism, insulin sensitivity, and body composition," Diabetes, vol. 62, no. 4, pp. 1186-1195, 2013. 


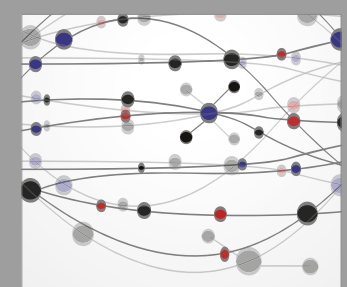

The Scientific World Journal
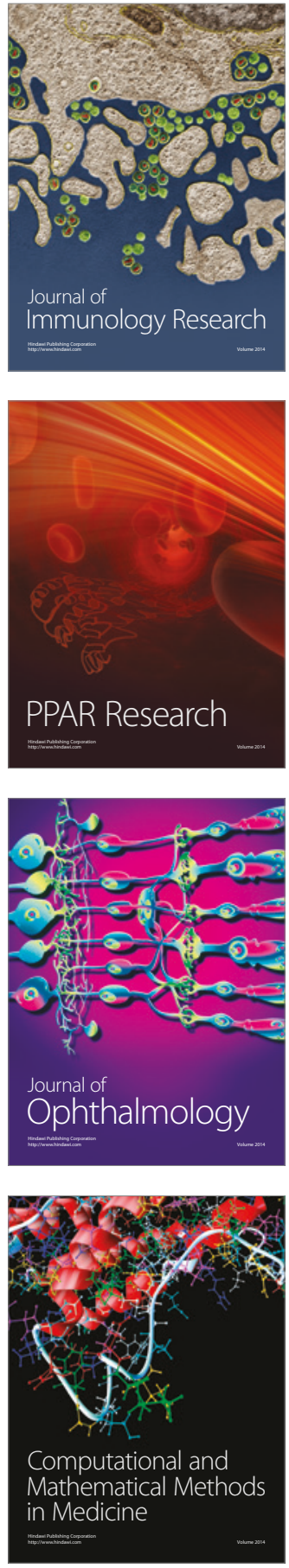

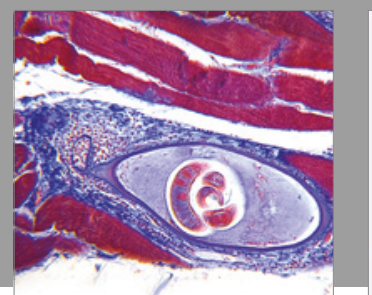

Gastroenterology Research and Practice
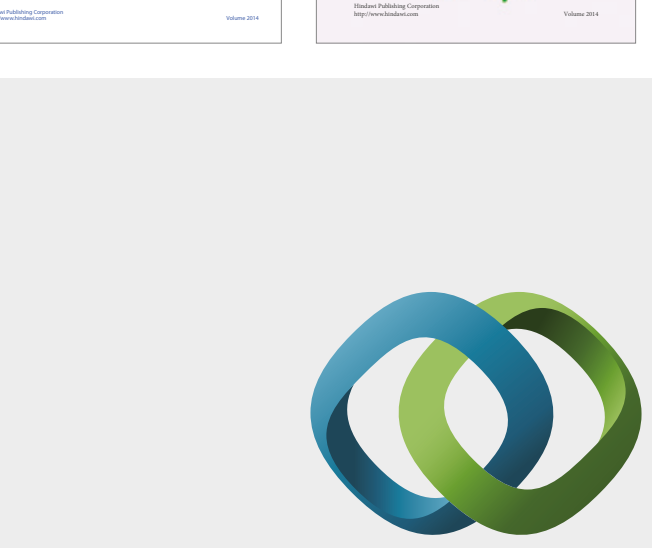

\section{Hindawi}

Submit your manuscripts at

https://www.hindawi.com
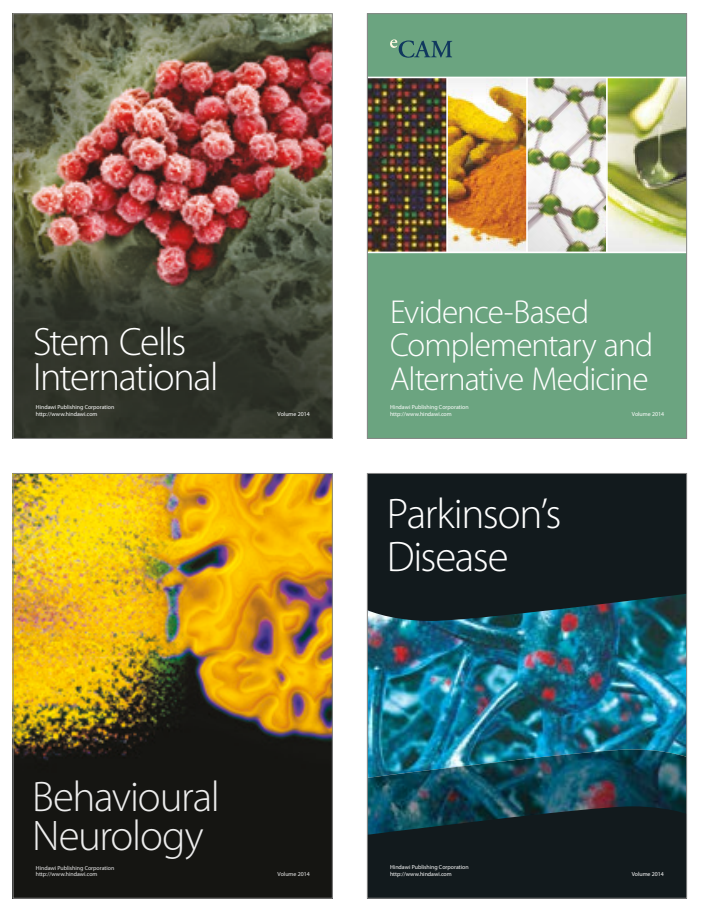
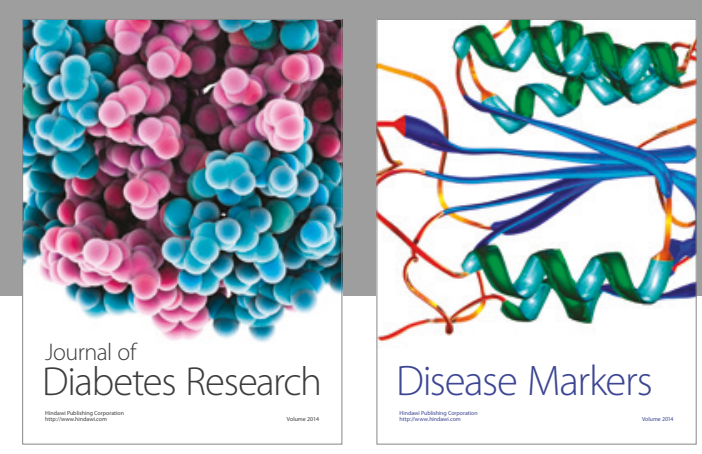

Disease Markers
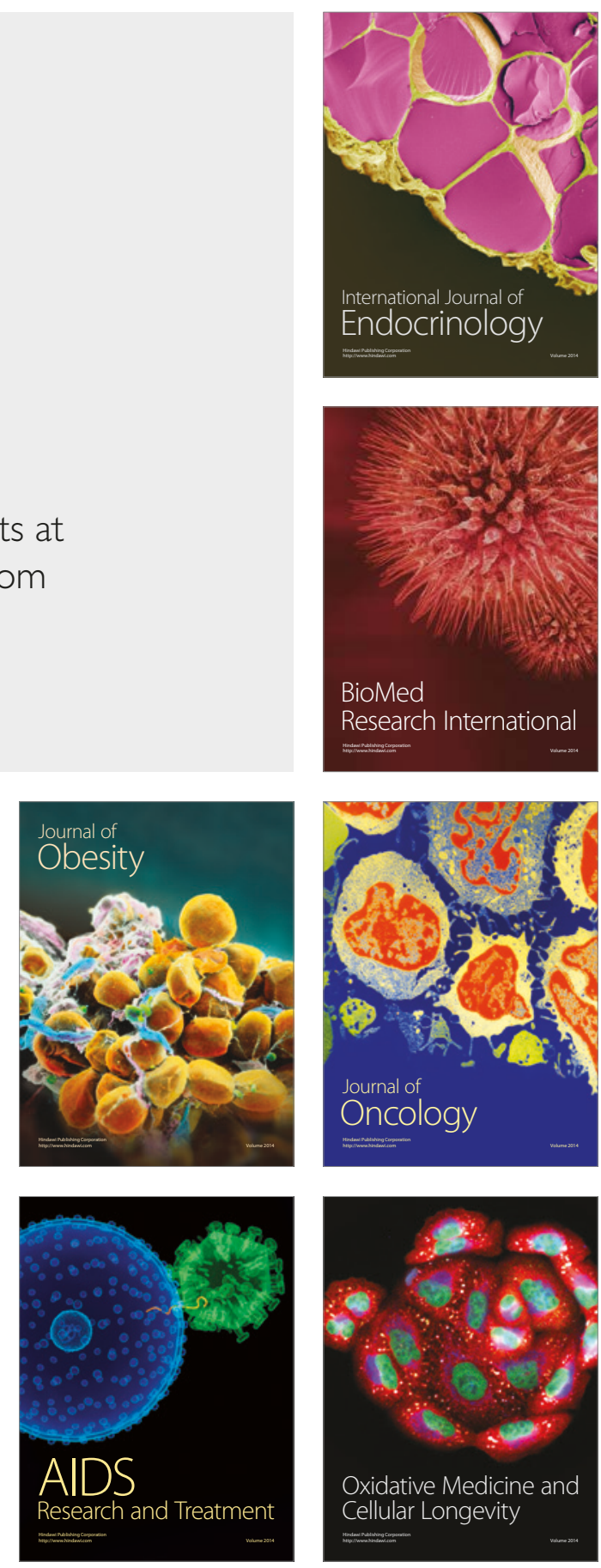